\title{
Article
}

\section{A new representation of extended Mittage-Leffler function and its properties}

\author{
Abdul Ghaffar ${ }^{1 *}$, Ayesha Saif ${ }^{1}$ and Faheem Khan ${ }^{2}$ \\ 1 Department of Mathematical Science, BUITEMS Quetta, Pakistan; abdulghaffar.jaffar@gmail.com(A. \\ Ghaffar) \\ 1 Department of Mathematical Science, BUITEMS Quetta, Pakistan; ayesha.saif@buitms.edu.pk \\ 2 Department of Mathematics, University of Sargodha, Pakistan \\ * Correspondence: abdulghaffar.jaffar@gmail.com; Tel.: +92-3347138562 \\ $\ddagger$ These authors contributed equally to this work. \\ Academic Editor: name \\ Version April 16, 2019 submitted to Preprints
}

$1 \quad$ Abstract: In this article, our main purpose is to establish a new extension of Mittag-Leffler function 2 by using the known extended beta function $\mathbf{B}_{\omega}(a, b ; p)$ introduced in [1]. It led to a novel extension of 3 the applicability of Mittag-Leffler function that introduced them as distributions defined for a specific 4 set of functions. We also, investigate some of its important properties, namely recursion relation,

5 Mellin transform and differential formulas.

$6 \quad$ Keywords: Mittag-Leffler function; extended Mittag-Leffler function; Extended beta function; Fox 7 Wright function

\section{Introduction}

Special functions are particular mathematical functions which are vital tools in higher calculus, and generally play a key role in almost all branches of applied and pure mathematics. Many well-known special functions are helpful to solve boundary value problems. In addition, solutions of many differential equations come out in terms of special functions. The zoo of special function contains Gamma function, Beta function, Hypergeometric function, Bessel's function, Mittag-Leffler function and many more. Special functions have been one of the most popular area of mathematics. In $17^{\text {th }}$ century, beta function was investigated by Legendre and Euler. Beta function is commonly used in probability theory, physics, engineering and other areas of mathematics.

Due to various implementations of generalized hypergeometric and Mittag-Leffler functions, many researchers have made their contribution to extend it in various forms. Recently, many authors [2-11] introduced several extensions of the well-known special functions due to their vital importance in mathematical and functional analysis.

We start with the Gosta Mittag-Leffler function $\mathbf{E}_{v}(z)$ [12] represented by the following series as:

$$
\mathbf{E}_{v}(z)=\sum_{k=0}^{\infty} \frac{z^{k}}{\Gamma(v k+1)},(z, v \in \mathbb{C} ; \boldsymbol{R e}(v)>0) .
$$

23 A more general form of Mittag-Leffler function given by Wiman [13] has the following form

$$
\mathbf{E}_{v, \tau}(z)=\sum_{k=0}^{\infty} \frac{z^{k}}{\Gamma(v k+\tau)},(z, v, \tau \in \mathbb{C} ; \boldsymbol{R e}(v)>0, \boldsymbol{R e}(\tau)>0) .
$$


24

Throughout this article, we will denote by $\mathbb{C}, \mathbf{N}, \mathbf{Z}_{0}^{-}$, and $\mathbb{R}^{+}$the set of Complex numbers, natural numbers, non-positive integers and positive real numbers respectively. Prabhakar [14] established the generalization of $\mathbf{E}_{v, \tau}(z)$ in the following form

$$
\mathbf{E}_{v, \tau}^{\lambda}(z)=\sum_{k=0}^{\infty} \frac{(\lambda)_{k} z^{k}}{\Gamma(v k+\tau) k !},(z, v, \tau, \lambda \in \mathbb{C} ; \boldsymbol{R e}(v)>0, \boldsymbol{R e}(\tau)>0),
$$

27 where $(\lambda)_{k}$ represents the Pochhammer symbol [15] defined as follows

$$
\begin{array}{rlr}
(\lambda)_{k} & =\left\{\begin{array}{cr}
1 ; & k=0, \lambda \in \mathbb{C}-\{0\} \\
\lambda(\lambda+1) \ldots(\lambda+k-1) ; & k \in \mathbf{N}, \lambda \in \mathbb{C}
\end{array}\right. \\
& =\frac{\Gamma(\lambda+k)}{\Gamma(\lambda)} \quad \lambda \in \mathbb{C}-\mathbf{Z}_{0}^{-} .
\end{array}
$$

Generalized Mittag-Leffler function $\mathbf{E}_{v, \tau}^{\lambda, s}(z)$ introduced by Prajapati and Shukla [16] has the following form

$$
\mathbf{E}_{v, \tau}^{\lambda, s}(z)=\sum_{k=0}^{\infty} \frac{(\lambda)_{s k} z^{k}}{\Gamma(v k+\tau) k !},(z, v, \tau, \lambda \in \mathbb{C} ; \operatorname{Re}(v)>0, \boldsymbol{R e}(\tau)>0, \operatorname{Re}(\lambda)>0 ; s \in(0,1) \cup \mathbf{N}),
$$

where $(\lambda)_{s k}=\frac{\Gamma(\lambda+s k)}{\Gamma(\lambda)}$ represents the generalized Pochhammer symbol. Ozarslan and Yilmaz [17] extended the Mittag-Leffler function $\mathbf{E}_{v, \tau}^{\lambda, s}(z)$ in the following way

$$
\mathbf{E}_{v, \tau}^{\lambda, s}(z ; p)=\sum_{k=0}^{\infty} \frac{\mathbf{B}_{p}(\lambda+k, s-\lambda)}{\mathbf{B}(\lambda, s-\lambda)} \frac{(s)_{k}}{\Gamma(v k+\tau)} \frac{z^{k}}{k !},
$$

where $(z, v, \tau, \lambda \in \mathbb{C} ; \boldsymbol{R e}(v)>0, \boldsymbol{\operatorname { R e }}(\tau)>0, \boldsymbol{\operatorname { R e }}(\lambda)>0 ; \boldsymbol{\operatorname { R e }}(p)>0, p=0 ; \boldsymbol{\operatorname { R e }}(s)>\operatorname{Re}(\lambda)>0)$ and

$$
\mathbf{B}_{p}(a, b)=\int_{0}^{1} u^{a-1}(1-u)^{b-1} \exp \left(-\frac{p}{u(1-u)}\right) d u,(\boldsymbol{R e}(p)>0, \boldsymbol{R} \mathbf{e}(a)>0, \boldsymbol{R} \mathbf{e}(b)>0) .
$$

is the extended Euler's beta function [18] and $\mathbf{B}_{0}(a, b)=\mathbf{B}(a, b)$ where $\mathbf{B}(a, b)$ is the classical beta function [15]. An interesting extension of extended beta function $\mathbf{B}_{p}(a, b)$ introduced by Parmar et al. [1] is of the following form

$$
\mathbf{B}_{\omega}(a, b ; p)=\sqrt{\frac{2 p}{\pi}} \int_{0}^{1} u^{a-\frac{3}{2}}(1-u)^{b-\frac{3}{2}} \mathbf{K}_{\omega+\frac{1}{2}}\left(\frac{p}{u(1-u)}\right) d u,(\omega \in \mathbb{C}, \boldsymbol{R e}(p)>0, \operatorname{Re}(a)>0, \operatorname{Re}(b)>0)
$$

where $\mathbf{K}_{\omega+\frac{1}{2}}$ is the modified Bessel's function. Motivated essentially by the demonstrated potential for applications of these generalized Mittag-Leffler functions in many diverse areas of mathematical, physical, engineering and statistical sciences, we introduce here another interesting extension of the extended Mittag-Leffler function as follows

$$
\mathbf{E}_{v, \tau}^{\lambda, s ; \omega}(z ; p)=\sum_{k=0}^{\infty} \frac{\mathbf{B}_{\omega}(\lambda+k, s-\lambda ; p)}{\mathbf{B}(\lambda, s-\lambda)} \frac{(s)_{k}}{\Gamma(v k+\tau)} \frac{z^{k}}{k !},
$$

where $(z, v, \tau, \lambda, \omega \in \mathbb{C} ; \boldsymbol{R} \mathbf{e}(v)>0, \boldsymbol{R e}(\tau)>0, \operatorname{Re}(\lambda)>0 ; \boldsymbol{R e}(p)>0 ; \operatorname{Re}(s)>\operatorname{Re}(\lambda)>0)$. For our ambition, we remember the Fox- Wright function ${ }_{r} \Psi_{S}[19]$

$$
{ }_{r} \Psi_{s}(z)=\sum_{k=0}^{\infty} \frac{\Gamma\left(\alpha_{1}+\tau_{1} k\right) \ldots \Gamma\left(\alpha_{r}+\tau_{r} k\right)}{\Gamma\left(\beta_{1}+\mu_{1} k\right) \ldots \Gamma\left(\beta_{s}+\mu_{s} k\right)} \frac{z^{k}}{k !}
$$


42 where $\tau_{i}$ and $\mu_{m} \in \mathbb{R}^{+}$such that

$$
1+\sum_{m=1}^{s} \mu_{m}-\sum_{i=1}^{r} \tau_{i}>0
$$

43 Further, making use of the extended Mittag-Leffler function (8). For each of these new extensions we

44 obtain various integral representations, properties and Mellin transforms, together with differentiation,

45 transformation, summation, generating function and asymptotic formulas.

46 1. Integral Formulae

${ }_{47} \quad$ In this section, we obtain several integral representations of Mittag-Leffler function $\left(\mathbf{E}_{v, \tau}^{\lambda, s ; \omega}(z ; p)\right)$

48 and consider certain special cases.

49 $\quad$ Theorem 1. Let $s, v, \tau, \lambda, \in \mathbb{C}$ with $\mathbf{R e}(s)>\boldsymbol{R e}(\lambda)>0, \boldsymbol{R e}(v)>0, \boldsymbol{R e}(\tau)>0$, and let $\operatorname{Re}(p)>0$. Then

$$
\begin{aligned}
\mathbf{E}_{v, \tau}^{\lambda, s ; \omega}(z ; p)= & \frac{1}{\mathbf{B}(\lambda, s-\lambda)} \sqrt{\frac{2 p}{\pi}} \times \\
& \int_{0}^{1}\left(u^{\lambda-\frac{3}{2}}(1-u)^{s-\lambda-\frac{3}{2}} \mathbf{K}_{\omega+\frac{1}{2}}\left(\frac{p}{u(1-u)}\right) \mathbf{E}_{v, \tau}^{s}(u z)\right) d u .
\end{aligned}
$$

50 Proof. By using equation (7) in (8), we get

$$
\begin{aligned}
\mathbf{E}_{v, \tau}^{\lambda, s ; \omega}(z ; p)= & \sum_{k=0}^{\infty}\left[\sqrt{\frac{2 p}{\pi}} \int_{0}^{1} u^{\lambda+k-\frac{3}{2}}(1-u)^{s-\lambda-\frac{3}{2}} \mathbf{K}_{\omega+\frac{1}{2}}\left(\frac{p}{u(1-u)}\right) d u\right. \\
& \left.\frac{(s)_{k}}{\mathbf{B}(\lambda, s-\lambda) \Gamma(v k+\tau)} \frac{z^{k}}{k !}\right] .
\end{aligned}
$$

51 After interchanging the order of integration and summation in the above equation which is justified 52 under the supposition of theorem (1), we obtain

$$
\begin{aligned}
\mathbf{E}_{v, \tau}^{\lambda, s ; \omega}(z ; p)= & \frac{1}{\mathbf{B}(\lambda, s-\lambda)} \sqrt{\frac{2 p}{\pi}} \int_{0}^{1}\left(u^{\lambda-\frac{3}{2}}(1-u)^{s-\lambda-\frac{3}{2}} \mathbf{K}_{\omega+\frac{1}{2}}\left(\frac{p}{u(1-u)}\right)\right. \\
& \left.\sum_{k=0}^{\infty} \frac{(s)_{k}}{\Gamma(v k+\tau)} \frac{(z u)^{k}}{k !}\right) d u .
\end{aligned}
$$

53 With the help of equation (3) in above equation, we attain the desired consequence.

$54 \quad$ Now, we obtain two different integral formulae of the extended MLF (10) as corollaries.

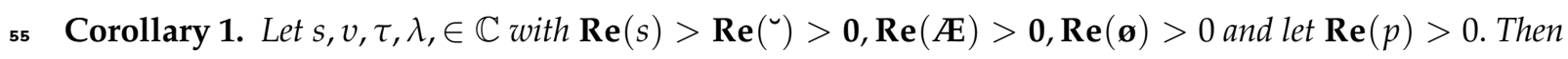

$$
\begin{aligned}
\mathbf{E}_{v, \tau}^{\lambda, s ; \omega}(z ; p)= & \frac{1}{\mathbf{B}(\lambda, s-\lambda)} \sqrt{\frac{2 p}{\pi}} \int_{0}^{\infty}\left[y^{\lambda-\frac{3}{2}}(1+y)^{1-s} \mathbf{K}_{\omega+\frac{1}{2}}\left(\frac{p(1+y)^{2}}{y}\right)\right. \\
& \left.\mathbf{E}_{v, \tau}^{s}\left(\frac{y z}{1+y}\right)\right] d y .
\end{aligned}
$$

56 Proof. If we set $u=\frac{y}{1+y}$, in theorem (1),we acquire the consequence we wish to prove. 


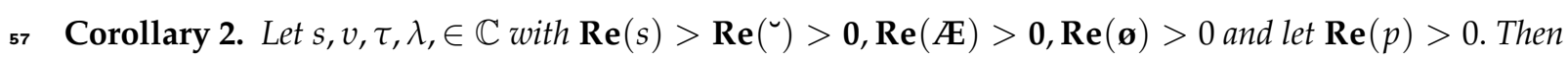

$$
\begin{aligned}
\mathbf{E}_{v, \tau}^{\lambda, s ; \omega}(z ; p)= & \frac{2}{\mathbf{B}(\lambda, s-\lambda)} \sqrt{\frac{2 p}{\pi}} \int_{0}^{\frac{\pi}{2}}\left[(\sin \psi)^{2(\lambda-1)}(\cos \psi)^{2(s-\lambda-1)}\right. \\
& \left.\mathbf{K}_{\omega+\frac{1}{2}}\left(\frac{p}{\sin ^{2} \psi \cos ^{2} \psi}\right) \mathbf{E}_{v, \tau}^{s}\left(z \sin ^{2} \psi\right)\right] d y
\end{aligned}
$$

58 Proof. If we set $u=\sin ^{2} \psi$, in theorem (1), we acquire the consequence we want to prove.

59 2. Mellin Transform Formula

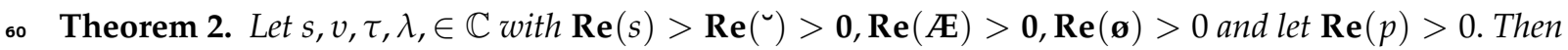

61 the Mellin ransform formula for the extended MLF (??) is

$$
\begin{aligned}
\mathbb{M}\left(\mathbf{E}_{v, \tau}^{\lambda, s ; \omega}(z ; p) ; r\right)= & \frac{2^{r-1}}{\sqrt{\pi}} \frac{\Gamma(s+r-\lambda)}{\Gamma(\lambda) \Gamma(s-\lambda)} \Gamma\left(\frac{r-\omega}{2}\right) \Gamma\left(\frac{r+\omega+1}{2}\right) \times \\
& { }_{2} \Psi_{2}\left[\begin{array}{l}
(s, 1),(\lambda+r, 1) ; \\
(\tau, v),(2 r+s, 1) ;
\end{array}\right] .
\end{aligned}
$$

62 Proof. By definition, Mellin transform of the MLF is

$$
\mathbb{M}\left(\mathbf{E}_{v, \tau}^{\lambda, s ; \omega}(z ; p) ; r\right)=\int_{0}^{\infty} p^{r-1} \mathbf{E}_{v, \tau}^{\lambda, s ; \omega}(z ; p) d p
$$

63 From equation (10), we have

$$
\begin{aligned}
\mathbb{M}\left(\mathbf{E}_{v, \tau}^{\lambda, s ; \omega}(z ; p) ; r\right)= & \int_{0}^{\infty}\left[p^{r-1} \frac{1}{\mathbf{B}(\lambda, s-\lambda)} \sqrt{\frac{2 p}{\pi}} \int_{0}^{1} u^{\lambda-\frac{3}{2}}(1-u)^{s-\lambda-\frac{3}{2}}\right. \\
& \left.\mathbf{K}_{\omega+\frac{1}{2}}\left(\frac{p}{u(1-u)}\right) \mathbf{E}_{v, \tau}^{s}(u z) d u\right] d p .
\end{aligned}
$$

${ }_{64}$ By interchanging the order of integration in equation (14) that is verified by the assumptions of this

65 theorem, we get

$$
\begin{aligned}
\mathbb{M}\left(\mathbf{E}_{v, \tau}^{\lambda, s ; \omega}(z ; p) ; r\right)= & \frac{1}{\mathbf{B}(\lambda, s-\lambda)} \sqrt{\frac{2}{\pi}} \int_{0}^{1}\left[u^{\lambda-\frac{3}{2}}(1-u)^{s-\lambda-\frac{3}{2}} \mathbf{E}_{v, \tau}^{\mathcal{s}}(u z)\right] \\
& \int_{0}^{\infty} p^{r-\frac{1}{2}} \mathbf{K}_{\omega+\frac{1}{2}}\left(\frac{p}{u(1-u)}\right) d p d u
\end{aligned}
$$

66 By putting $y=\frac{p}{u(1-u)}$ in the internal integral in equation (15), we have

$$
\begin{aligned}
\mathbb{M}\left(\mathbf{E}_{v, \tau}^{\lambda, s ; \omega}(z ; p) ; r\right)= & \frac{1}{\mathbf{B}(\lambda, s-\lambda)} \sqrt{\frac{2}{\pi}} \int_{0}^{1} u^{\lambda+r-1}(1-u)^{s+r-\lambda-1} \mathbf{E}_{v, \tau}^{s}(u z) \\
& {\left[\int_{0}^{\infty} y^{r-\frac{1}{2}} \mathbf{K}_{\omega+\frac{1}{2}}(y) d y\right] d u . }
\end{aligned}
$$

67 By using the consequence (Olver et al. (2010))

$$
\int_{0}^{\infty} y^{r-\frac{1}{2}} \mathbf{K}_{\omega+\frac{1}{2}}(y) d y=2^{r-\frac{3}{2}} \Gamma\left(\frac{r-\omega}{2}\right) \Gamma\left(\frac{r+\omega+1}{2}\right),
$$


68 and then applying definition (9) in equation (16), we have

$$
\begin{aligned}
\mathbb{M}\left(\mathbf{E}_{v, \tau}^{\lambda, s ; \omega}(z ; p) ; r\right)= & \frac{1}{\mathbf{B}(\lambda, s-\lambda)} \frac{2^{r-1}}{\sqrt{\pi}} \Gamma\left(\frac{r-\omega}{2}\right) \Gamma\left(\frac{r+\omega+1}{2}\right) \\
& \int_{0}^{1} u^{\lambda+r-1}(1-u)^{s+r-\lambda-1} \sum_{k=0}^{\infty} \frac{(s)_{k}}{\Gamma(v k+\tau)} \frac{(u z)^{k}}{k !} d u .
\end{aligned}
$$

69 By interchanging the order of summation and integration and then applying the definition (2) in equation (17), we obtain

$$
\begin{aligned}
\mathbb{M}\left(\mathbf{E}_{v, \tau}^{\lambda, s ; \omega}(z ; p) ; r\right)= & \frac{2^{r-1}}{\sqrt{\pi}} \frac{\Gamma(s+r-\lambda)}{\Gamma(\lambda) \Gamma(s-\lambda)} \Gamma\left(\frac{r-\omega}{2}\right) \Gamma\left(\frac{r+\omega+1}{2}\right) \\
& \sum_{k=0}^{\infty} \frac{\Gamma(s+k) \Gamma(\lambda+r+k)}{\Gamma(\tau+v k) \Gamma(2 r+s+k)} \frac{z^{k}}{k !} .
\end{aligned}
$$

71 By the implementation of equation (9) in equation (18), we obtain the required consequence.

72

7 the derivative formula for the extended MLF (8) is

$$
\frac{d^{k}}{d z^{k}} \mathbf{E}_{v, \tau}^{\lambda, s ; \omega}(z ; p)=(\lambda)_{k} \mathbf{E}_{v, k v+\tau}^{\lambda+k, s+k ; \omega}(z ; p), \quad(k \in \mathbb{N} \cup\{0\}) .
$$

78 Proof. From equation (8), we have

$$
\begin{aligned}
\frac{d}{d z} \mathbf{E}_{v, \tau}^{\lambda, s ; \omega}(z ; p) & =\sum_{k=1}^{\infty}\left(\frac{\mathbf{B}_{\omega}(\lambda+k, s-\lambda ; p)}{\mathbf{B}(\lambda, s-\lambda)} \frac{(s)_{k}}{\Gamma(v k+\tau)} \frac{z^{k-1}}{(k-1) !}\right) \\
& =s \sum_{k=0}^{\infty}\left(\frac{\mathbf{B}_{\omega}(\lambda+1+k, s-\lambda ; p)}{\mathbf{B}(\lambda, s-\lambda)} \frac{(s+1)_{k}}{\Gamma(v k+v+\tau)} \frac{z^{k}}{k !}\right) .
\end{aligned}
$$

79 Using (5), we obtain

$$
\frac{d}{d z} \mathbf{E}_{v, \tau}^{\lambda, s ; \omega}(z ; p)=\lambda \sum_{k=0}^{\infty}\left(\frac{\mathbf{B}_{\omega}(\lambda+1+k, s-\lambda ; p)}{\mathbf{B}(\lambda+1, s-\lambda)} \frac{(s+1)_{k}}{\Gamma(v k+v+\tau)} \frac{z^{k}}{k !}\right) .
$$

so In terms of (8), we get

$$
\frac{d}{d z} \mathbf{E}_{v, \tau}^{\lambda, s ; \omega}(z ; p)=\lambda \mathbf{E}_{v, v+\tau}^{\lambda+1, s+1 ; \omega}(z ; p)
$$


81 Similarly,we have

$$
\frac{d^{2}}{d z^{2}} \mathbf{E}_{v, \tau}^{\lambda, s ; \omega}(z ; p)=(\lambda)_{2} \mathbf{E}_{v, 2 v+\tau}^{\lambda+2, s+2 ; \omega}(z ; p)
$$

82 By taking $n$th order derivative of the MLF (9), we obtain the desired result.

83 Theorem 4. Let $s, v, \tau, \lambda$, and $\eta \in \mathbb{C}$ with $\mathbf{R e}(s)>\mathbf{R e}(\lambda)>0, \boldsymbol{R e}(v)>0, \mathbf{R e}(\tau)>0$ and let $\mathbf{R e}(p)>0$.

84 Then

$$
\frac{d^{k}}{d z^{k}}\left(z^{\tau-1} \mathbf{E}_{v, \tau}^{\lambda, s ; \omega}\left(\eta z^{v} ; p\right)\right)=z^{\tau-k-1} \mathbf{E}_{v, \tau}^{\lambda, s ; \omega}\left(\eta z^{v} ; p\right), \quad(k \in \mathbb{N} \cup\{0\})
$$

85 Proof. From equation (8), we get

$$
\begin{aligned}
\frac{d}{d z}\left[z^{\tau-1} \mathbf{E}_{v, \tau}^{\lambda, s ; \omega}\left(\eta z^{v} ; p\right)\right]= & \frac{d}{d z} \sum_{k=0}^{\infty}\left[\frac{\mathbf{B}_{\omega}(\lambda+k, s-\lambda ; p)}{\mathbf{B}(\lambda, s-\lambda)} \frac{(s)_{k}}{\Gamma(v k+\tau)}(v k+\tau-1)\right. \\
& \left.\frac{\eta^{k} z^{v k+\tau-1}}{k !}\right] \\
= & z^{\tau-2} \sum_{k=0}^{\infty} \frac{\mathbf{B}_{\omega}(\lambda+k, s-\lambda ; p)}{\mathbf{B}(\lambda, s-\lambda)} \frac{(s)_{k}}{\Gamma(v k+\tau-1)} \frac{\left(\eta z^{v}\right)^{k}}{k !}
\end{aligned}
$$

86 By using equation (8), above equation can be written as

$$
\frac{d}{d z}\left[z^{\tau-1} \mathbf{E}_{v, \tau}^{\lambda, s ; \omega}\left(\eta z^{v} ; p\right)\right]=z^{\tau-2} \mathbf{E}_{v, \tau-1}^{\lambda, s ; \omega}\left(\eta z^{\nu} ; p\right)
$$

87 Similarly, we have

$$
\frac{d^{2}}{d z^{2}}\left[z^{\tau-1} \mathbf{E}_{v, \tau}^{\lambda, s ; \omega}\left(\eta z^{\nu} ; p\right)\right]=z^{\tau-3} \mathbf{E}_{v, \tau-2}^{\lambda, s ; \omega}\left(\eta z^{v} ; p\right)
$$

s8 After differentiating equation (8) $k$ times, we get the desired result.

89 4. Recurrence Relation

9o Theorem 5. Let $s, v, \tau, \lambda, \in \mathbb{C}$ with $\mathbf{R e}(s)>\operatorname{Re}(\lambda)>0, \boldsymbol{R e}(v)>0, \boldsymbol{R e}(\tau)>0$ and let $\operatorname{Re}(p)>0$, then

$$
\mathbf{E}_{v, \tau}^{\lambda, s ; \omega}(z ; p)=\tau \mathbf{E}_{v, \tau+1}^{\lambda, s ; \omega}(z ; p)+v s z \mathbf{E}_{v, v+\tau+1}^{\lambda+1, s+1 ; \omega}(z ; p) .
$$

91 Proof. By using (8) in the integrand of (10), we get

$$
\begin{aligned}
\mathbf{E}_{v, \tau}^{\lambda, s ; \omega}(z ; p)= & \frac{1}{\mathbf{B}(\lambda, s-\lambda)} \sqrt{\frac{2 p}{\pi}} \int_{0}^{1}\left[u^{\lambda-\frac{3}{2}}(1-u)^{s-\lambda-\frac{3}{2}} \mathbf{K}_{\omega+\frac{1}{2}}\left(\frac{p}{u(1-u)}\right)\right. \\
& \left.\tau \mathbf{E}_{v, \tau+1}^{s}(u z)\right] d u+\frac{1}{\mathbf{B}(\lambda, s-\lambda)} \sqrt{\frac{2 p}{\pi}} \int_{0}^{1}\left[u^{\lambda-\frac{3}{2}}(1-u)^{s-\lambda-\frac{3}{2}}\right. \\
& \left.\mathbf{K}_{\omega+\frac{1}{2}}\left(\frac{p}{u(1-u)}\right) v z \frac{d}{d z} \mathbf{E}_{v, \tau+1}^{s}(u z)\right] d u
\end{aligned}
$$


92

\section{${ }_{95}$ References}

96 191-201.

Using equation (21) for $k=1$ in equation (23), we get

$$
\begin{aligned}
\mathbf{E}_{v, \tau}^{\lambda, s ; \omega}(z ; p)= & \tau \mathbf{E}_{v, \tau+1}^{\lambda, s ; \omega}(z ; p)+v s z \frac{1}{\mathbf{B}(\lambda, s-\lambda)} \sqrt{\frac{2 p}{\pi}} \\
& \int_{0}^{1}\left[u^{(\lambda+1)-\frac{3}{2}}(1-u)^{(s+1)-(\lambda+1)-\frac{3}{2}}\right. \\
& \left.\mathbf{K}_{\omega+\frac{1}{2}}\left(\frac{p}{u(1-u)}\right) \mathbf{E}_{v, v+\tau+1}^{s+1}(u z)\right] d u .
\end{aligned}
$$

Using equation (10) in above equation, we obtain

$$
\mathbf{E}_{v, \tau}^{\lambda, s ; \omega}(z ; p)=\tau \mathbf{E}_{v, \tau+1}^{\lambda, s ; \omega}(z ; p)+v s z \mathbf{E}_{v, v+\tau+1}^{\lambda+1, s+1 ; \omega}(z ; p)
$$

1. Parmar, R. K., Chopra, P. U. R. N. I. M. A., \& Paris, R. B. (2015). On an extension of extended beta and hypergeometric functions. Journal of Classical Analysis Volume 11, Number 2 (2017), 91-106.

2. Chaudhry, M. A., \& Zubair, S. M. (1994). Generalized incomplete gamma functions with applications. Journal of Computational and Applied Mathematics, 55(1), 99-123.

3. Chaudhry, M. A., \& Zubair, S. M. (1995). On the decomposition of generalized incomplete gamma functions with applications to Fourier transforms. Journal of Computational and Applied Mathematics, 59(3), 253-284.

4. Chaudhry, M. A., Temme, N. M., \& Veling, E. J. M. (1996). Asymptotics and closed form of a generalized incomplete gamma function. Journal of Computational and Applied Mathematics, 67(2), 371-379.

5. Chaudhry, M. A., Qadir, A., Rafique, M., \& Zubair, S. M. (1997). Extension of Euler's beta function. Journal of Computational and Applied Mathematics, 78(1), 19-32.

6. Chaudhry, M. A., \& Zubair, S. M. (2002). Extended incomplete gamma functions with applications. Journal of Mathematical Analysis and Applications, 274(2), 725-745.

7. Chaudhry, M. A., Qadir, A., Srivastava, H. M., \& Paris, R. B. (2004). Extended hypergeometric and confluent hypergeometric functions. Applied Mathematics and Computation, 159(2), 589-602

8. Rahman G, Ghaffar A, Mubeen S, Arshad M, Khan SU. The composition of extended Mittag-Leffler functions with pathway integral operator. Advances in Difference Equations. 2017 Dec;2017(1):176.

9. Rahman G, Ghaffar A, Nisar KS, Azeema A. The (k, s)-Fractional Calculus of Class of a Function. Honam Mathematical J. 40 (2018), No. 1, pp. 125-138 https:/ / dx.doi.org/10.5831/HMJ.2018.40.1.125

10. Rahman G, Ghaffar A, Nisar KS, Mubeen S. A New Class of Integrals Involving Extended Mittag-Leffler Functions. Journal of Fractional Calculus and Applications (JFCA), 8(20) 56-61, 2017

11. Araci, S., Rahman, G., Ghaffar, A., \& Nisar, K. S. (2019). Fractional Calculus of Extended Mittag-Leffler Function and Its Applications to Statistical Distribution. Mathematics, 7(3), 248.

12. Mittag-Leffler, G. M. (1903). Sur la nouvelle fonction Ea (x). CR Acad. Sci. Paris, 137(2), 554-558.

13. Wiman, A. (1905). Über den Fundamentalsatz in der Teorie der FunktionenE a (x). Acta Mathematica, 29(1),

14. Prabhakar, T. R. (1971). A singular integral equation with a generalized Mittag Leffler function in the kernel.

15. Srivastava, H. M., and Choi, J. (2011). Zeta and q-Zeta functions and associated series and integrals. Elsevier.

16. Shukla, A. K., and Prajapati, J. C. (2007). On a generalization of Mittag-Leffler function and its properties. Journal of Mathematical Analysis and Applications, 336(2), 797-811.

17. Ozarslan, M. A., and Yilmaz, B. (2014). The extended Mittag-Leffler function and its properties. Journal of Inequalities and Applications, 2014(1), 85.

18. Chaudhry, M. A., Qadir, A., Srivastava, H. M., and Paris, R. B. (2004). Extended hypergeometric and confluent hypergeometric functions. Applied Mathematics and Computation, 159(2), 589-602.

19. Fox, C. (1928). The asymptotic expansion of generalized hypergeometric functions. Proceedings of the London Mathematical Society, 2(1), 389-400. 